\title{
Where is the contre-coup? Atypical localization of occipital brain contusion
}




\title{
Where is the contre-coup? Atypical localization of occipital brain contusion
}

\author{
A.I. Cucu ${ }^{1}$, Claudia Florida Costea ${ }^{1,2}$, R.A. Sascau ${ }^{2,3}$, B. Dobrovat ${ }^{1,2}$, \\ I. Poeata ${ }^{1,2}$, Turliuc Serban ${ }^{2}$, Dana Mihaela Turliuc ${ }^{1,2}$ \\ 1"Prof. Dr. N. Oblu" Emergency Clinical Hospital, Iași, ROMANIA \\ 2"Grigore T. Popa" University of Medicine and Pharmacy, Iași, ROMANIA \\ 3"George I. M. Georgescu" Institute of Cardiovascular Diseases, Iasi, ROMANIA
}

\begin{abstract}
Most traumatic brain injuries with occipital impact cause frontal and temporal contre-coup lesions, while patients with coagulopathies are more susceptible to such lesions. We present the case of a chronic ethylic patient with thrombocytopenia, who was treated for ethanol withdrawal syndrome at a psychiatric service. The head CT scan revealed an occipital contusion with atypical localization and the absence of frontotemporal contre-coup lesions. In conclusion, it is recommended a head CT scan to chronic alcoholic patients with psychiatric manifestations, especially in the context of systemic coagulopathies related to excessive alcohol consumption. In the case of chronic ethylic patients that frequently display systemic coagulation disorders, the head CT scan is necessary at the first psychiatric manifestations.
\end{abstract}

Key words: atypical occipital contusion, brain injury, coagulopathy

\section{Introduction}

Traumatic brain injuries represent a major cause of morbidity and mortality worldwide (13). Traumatic parenchymal mass lesions are a common sequel of traumatic brain injury (TBI), with an incidence of up to $8.2 \%$ of all TBI (6) cases and $13-35 \%$ of all severe TBI cases (1). Out of these, brain contusions are one of the most common forms of TBI, and they occur most frequently in areas where the sudden deceleration of the head causes brain damage to bony prominences such as the frontal, temporal and occipital poles, in a manner known in literature as coup and contre-coup brain contusions. Originally described in the 17th century by French surgeon Jean Louis Petit (1674-1750) (9), our knowledge of these injuries was completed in 1766 by another French surgeon, Antoine Louis (1723-1792), who presented the results of his research at Académie Nationale de Médecine (4). Two years later, in 1768, coup and contrecoup lesions were better understood by the military surgeon of the same origin as his predecessors, Louis Sebastian Saucerotte (1741-1814), thanks to the experiments that he carried out on 28 dogs (12). 


\section{Short case report}

We present the case of a 55-year-old chronic ethylic patient hospitalized in the Department of Neurosurgery of "Prof. Dr. N. Oblu" Emergency Clinical Hospital, who was transferred from a psychiatric service due to neurological aggravation (GCS 13). The patient was admitted to the 24-hour psychiatric service for ethanol withdrawal syndrome with delirium. The anamnesis failed to identify, but allowed to suspect the existence of a minor TBI.

At the time of admission, the patient presented a GCS of 13 and petechiae at the level of the anterior and posterior thorax as well as on the limbs (Figure 1A) The performed head CT scan revealed a left occipital contusion associated with an occipital hemorrhage (Figure 1B, $C$ and Figure 2A, B - red arrows) and a left hemispheric acute subdural haematoma (Figure $1 \mathrm{C}$ and Figure 2A, B - blue arrows). The patient also had thrombocytopenia $(65,000 / \mathrm{mcL})$, elevated liver enzymes (TGP $100 \mathrm{U} / \mathrm{L}$, TGO $117 \mathrm{U} / \mathrm{L}$, GGT $307 \mathrm{U} / \mathrm{L}$ ), as well as dyselectrolymia. Surgery was performed, by evacuating the left occipital hemorrhage and the right subdural hemispheric haematoma through a left parietal-occipital approach (Figure 2C, D).
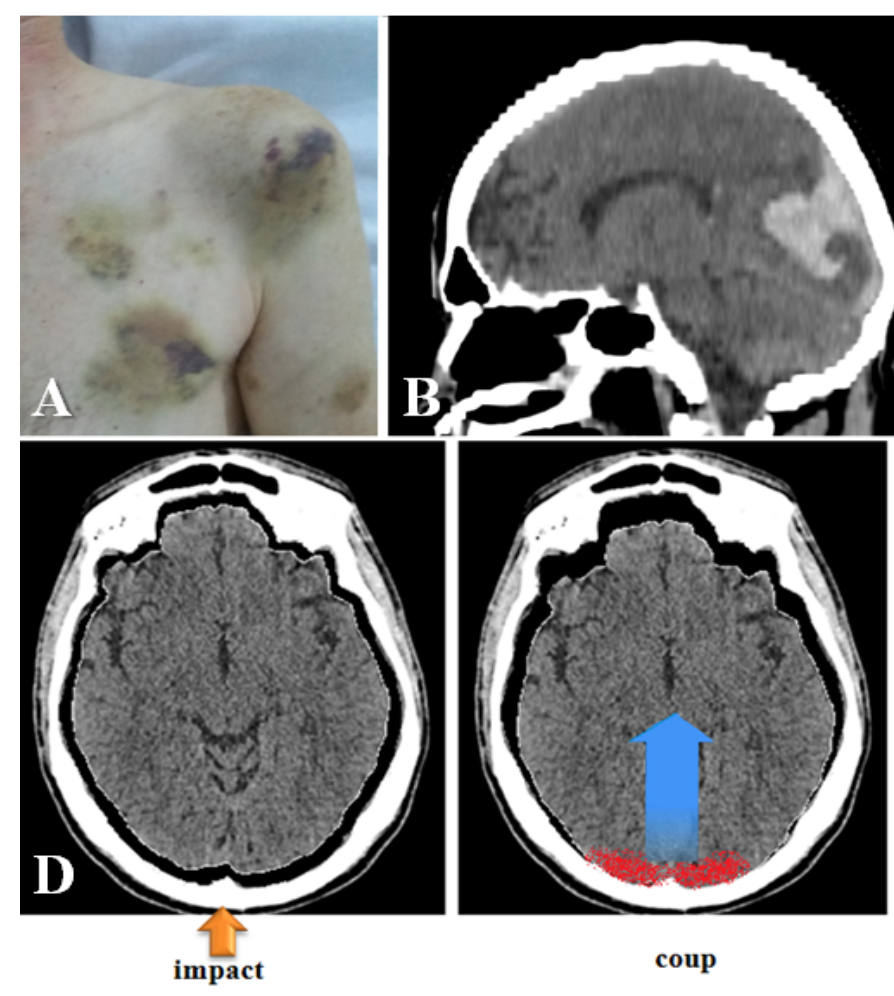
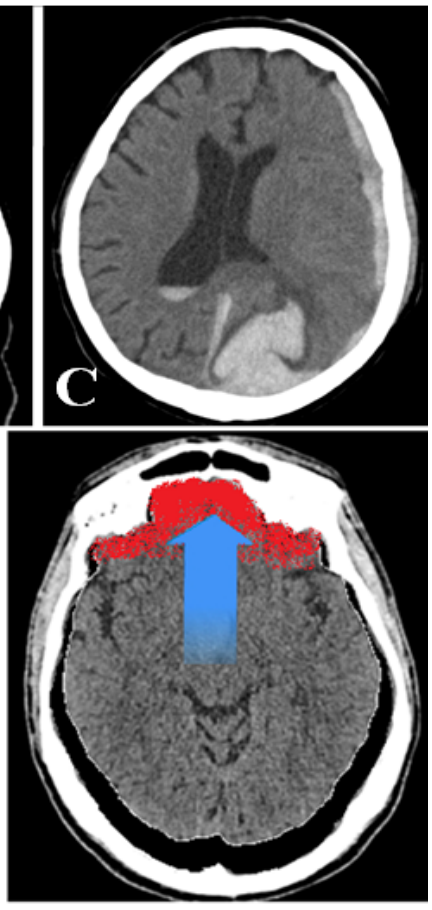

contrecoup

Figure 1 - Petechiae at the level of anterior thorax (A); Occipital brain contusion and hemorrhage (B) and left hemispheric acute subdural haematoma (C). Coup and counter-coup injuries in TBI with occipital impact (D)

(personal collection of authors) 

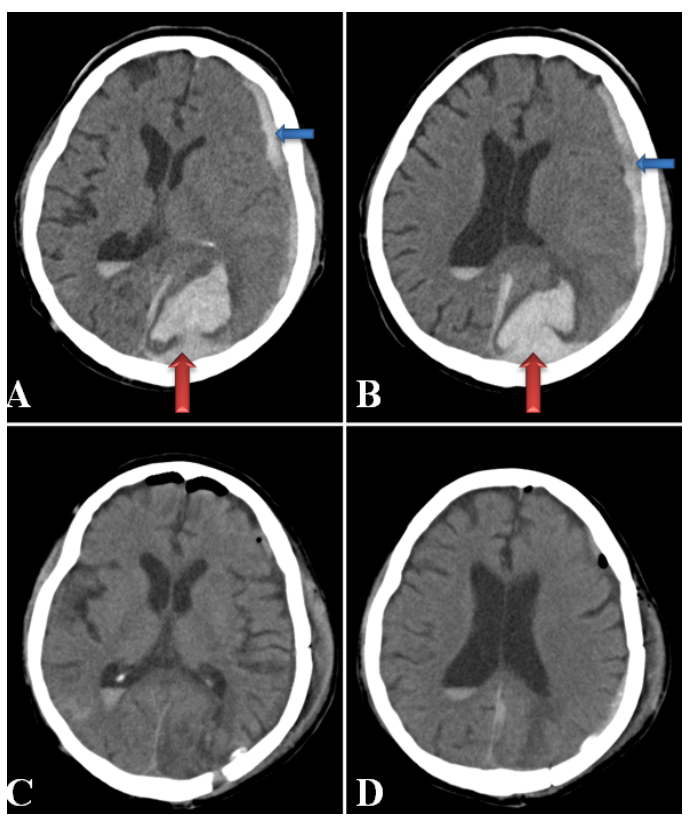

Figure 2 - Preoperative (A) and postoperative (B) imaging aspects

\section{Discussions}

In terms of brain contusions, clinical observations have indicated that in the absence of a skull fracture, most cerebral contusions occur in the frontal and temporal lobes, in the case of occipital impacts, and on the same side in most frontal impacts (2).

In TBI with occipital impact, the pattern of brain contusions varies depending on the location of the impact. In the case of midline occipital impacts, contre-coup lesions consist in localized bilateral frontal and temporal lobe contusions (Figure 1D). In the case of TBI with lateral occipital impact, contralateral contrecoup contusions are localized at the frontal and temporal lobes on the opposite side of the impact. Practically, in the case of TBI with occipital impact, these contre-coup lesions are more severe due to the rough edges of the sphenoid wings and the floor of the frontal anterior fossa, compared to occipital lobe injuries (8).

Usually, in the case of occipital impacts, contre-coup contusions are localized in the frontal and temporal lobes, whereas the coup contusions are primarily in the cerebellum, without affecting the occipital lobes. When occipital impacts fracture the occipital bone, the lesion distribution changes and coup lesions are predominant, being associated with the occipital lobes, while contre-coup lesions are smaller, although they have the same fronto-temporal localization (7).

It is well-known that coagulopathies, within or outside the context of a TBI, lead to intracranial hemorrhage, including thrombocytopenia induced by alcohol or liver disease, which are directly caused by the toxic effects of alcohol on the bone marrow and associated folate deficiency. In the case of our patient, his pre-existing biological context (coagulopathy due to alcoholic hepatopathy) favored, even in the case of a minor TBI, the bleeding of microvessels fractured at the time of primary injury, causing extravasation of blood with occipital hemorrhage. In addition to that, a number of hemostasis abnormalities are present in alcoholic patients, including numerous functional deficits in the platelets such as decreased platelet aggregation $(5,11)$ associated with disturbances in the ultrastructural morphology (3).

\section{Conclusion}

Head CT scan is mandatory even for chronic alcoholics known for psychiatric disorders, especially in case that, in the context 
of their condition, they are predisposed to contusions and cerebral haemorrhages as a consequence of minor trauma or even in the absence of such TBI.

\section{Correspondence}

\section{Claudia Florida Costea}

"Prof. Dr. N. Oblu" Emergency Clinical Hospital, Iași, Romania

E-mail:costea10@yahoo.com

\section{References}

1.Bullock MR, Chesnut R, Ghajar J, et al. Surgical management of traumatic parenchymal lesions. Neurosurgery. 2006; 58:S25-S46.

2.Chu CS, Lin MS, Huang HM, Lee MC. Finite element analysis of cerebral contusion. J Biomech. 1994; 27:187194.

3.Cowan DH. Effect of alcoholism on hemostasis. Semin Hematol. 1980; 17:137-147.

4.Finger S. Origins of neuroscience: a history of explorations into brain function. Oxford University Press, New York. 2001. p 429.
5.Haut MJ, Cowan DH. The effect of ethanol on hemostatic properties of human blood platelets. Am J Med. 1974; 56:22-33.

6.Mandera M, Zralek C, Krawczyk I, et. al. Surgery or conservative treatment in children with traumatic intracerebral haematoma. Childs Nerv Syst. 1999; 15:267269.

7.Overgaard J. Traumatic injuries of the planum occipitale and posterior fossa brain parenchyma. In Samii M, Brihaye (eds). Traumatology of the skull base: anatomy, clinical and radiological diagnosis operative treatment. Springer, Berlin. 2012. p 88-93.

8.Parker RS. Concussive brain trauma: neurobehavioral impairment and maladaptation, CRC Press, Boca Raton. 2016. p 217.

9.Petit JL. Oeuvres completes de Jean-Louis Petit. Chapoulaud F., 1837.

10.Quinones-Hinojosa A, Gulati M, Singh V, Lawton MT. Spontaneous intracerebral hemorrhage due to coagulation disorders. Neurosurg Focus. 2003; 15:E3.

11.Salem RO, Laposata M. Effects of alcohol on hemostasis. Am J Clin Pathol. 2005; 123 Suppl:S96-105. 12.Saucerotte LS. Mélanges de chirurgie Neapoli, Gay, Italy. 1801.

13.Turliuc D, Cucu A. Management of mild and moderate head injuries in adults. Romanian Neurosurgery. 2010; 17:421-431. 